\title{
The Latent Structure of the Center for Epidemiological Studies Depression Scale (CES-D) Among Older Adults in China
}

\author{
To-Han Chang ${ }^{1}$ and Fang Hua Jhang ${ }^{2, *}$ \\ ${ }^{1}$ Department of History, Minjiang University, Fuzhou, Fujian 350108, China \\ ${ }^{2}$ Department of Law and Social Work, Minjiang University, Fuzhou, Fujian 350108, China \\ *Corresponding author. Email: doubleclectic@yahoo.com.tw
}

\begin{abstract}
Little attention is paid to the factorial structure of (the Center for Epidemiological Studies Depression Scale, CES-D) among Chinese older adults. This study examined the latent structure of the short-form and full scale CES-D in older adults in China. A sample of 3770 older adults aged over 65 was derived from two waves of China Family Panel Studies. Confirmatory factor analysis (CFA) was employed to analyze the psychometric properties of the CES-D. The results of CFA showed that the four-factor model for the full scale and the three-factor model for the short-form scale demonstrated superior fit to other models. Thus, our findings provide evidence of acceptable measurement properties of the CES-D in Chinese older adults.
\end{abstract}

Keywords: CES-D; China; measurement properties; older adults

\section{INTRODUCTION}

China has the highest number of older adults in the world with a rapidly increasing aging population [1]. In 2019, China had approximately 176 million adults aged 65 and above, accounting for $12.6 \%$ of the total population [2]. It is expected to become an aged society prior to 2025 . Using the Center for Epidemiological Studies Depression Scale (CES-D), Qin, Wang and Hsieh [3] found that at least 30\% of Chinese adults suffered from depressive symptoms (a score of 16 as a threshold value) in 2012 and that older people are more likely to be depressed and that older people are more likely to be depressed. When Radloff [4] designed the CES-D, he measured depressive symptoms in a community-based population rather than in an older population. It remains unclear whether the scale captures the same phenomenon in China as the construct validity of the four-factor structure supported by many Western gerontology studies [5]. There are very few studies that explore the latent structure of the CES-D in older adult population in China. Only one study has examined the factor structure of the full scale in people aged 55 and over in China [6], but it did not test the two-factor model that is supported by previous studies. As depression to some extent is shaped by culture [7], it is essential to assess whether the scale measures the same construct in the same way as the Western studies. This will provide a foundation for comparison of measurement in variance across countries.

\subsection{The Factorial Structure of the CES-D in Elderly Populations and Chinese Adult Populations}

Radloff [4] used exploratory factor analysis (EFA) to identify a four-factor structure of the 20-item CES-D, including depressed affect, somatic symptoms, interpersonal problems, and positive affect, in a general population. More recent work has identified a range of two- and three-factor alternative structures of the scale in older adults and Chinese adults. The two-factor model (depressed affect and positive affect) is supported by earlier research [8], despite the fact that they did not measure all the items of the scale in Indian older adults. Cosco et al. [5] reviewed the factor structure of 20-item CES-D among older adults and found that older adults in Asian countries may express depression in a more somatically oriented way than their Western counterparts. A cross-culture study revealed that Chinese adults tend to report more somatic symptoms than Euro-Canadians [9]. In a Chinese context, where there is high value on expressing somatic symptoms and emotional control [9,10], the distinction between depressed affect and somatic symptoms may become unimportant. An alternative twofactor model of the full scale identified depressive symptoms and interpersonal problems in Hong Kong Chinese married couples, which reflects that Hong Kong Chinese tend to blur the differences between somatic symptoms and depressed affect [11]. A three-factor model was found to hold in a rural area in China, with depressed affect and somatic symptoms combined as one factor [12] Despite these variations, the extant literature largely 
supports the four-factor model of the full scale, in elderly populations [5,6]. Zhang et al. [6] compared a one-, threeand four-factor model and the second-order factor model and found that a four-factor model fitted the data best in older Chinese and Dutch samples.

Most researchers study the factorial structure of the full scale for the aged population, but there are very few studies that explore that of the short-form CES-D for older adults. Of these scales, the eight-item CES-D including depressed affect, somatic symptoms and positive affect is widely used in many large surveys [13]. Previous research provided support for a single factor structure for the eightitem CES-D in European older people, but the study did not test other models [13]. Although research on the latent structure of the CES-D 8 is limited, the three-factor model may get support from the extant literature on the full scale and short-form scale in elderly populations $[5,6,14]$. In a 10 -item abbreviated version of the CES-D that is the same as the eight-item version with two additional items about interpersonal problems, a four-factor structure has been identified in a group of older people [14]. Although it remains unknown whether the CES-D 8, as the full scale, demonstrates distinct dimensions in older Chinese adults, the three-factor model may demonstrate better model fit than other models in this short-form scale based on the extant studies.

In a nutshell, the study aims to examine the factorial structure of the CES-D among the elderly in China. It is expected that four-factor model in the full scale will fit the data better than alternative models in the older population. It is hypothesized that three-factor model in the short-form scale will fit the data better than alternative models among the older adults.

\section{METHOD}

\subsection{Sample}

The study used data from China Family Panel Studies (CFPS), a nationally representative sample of people aged 16 years and older. The CFPS adopted a stratified threestage (districts/counties-villages/communities-households) probability random sampling method to collect information regarding individual backgrounds, mental health and social capital. The survey was launched in 2010 , but the present study primarily focused on data regarding the CES-D collected in two later waves, 2012 (T1) and 2016 (T2). At $\mathrm{T} 1$ (overall response rate $74.1 \%$ ), data were selected from a sample of 4615 adults aged 65 years or above. At T2, data were available for 3770 of these 4615 respondents, yielding an acceptable attrition rate of $18.3 \%$. The missing rates for key items measuring depressive symptoms, LS and two types of trust ranged from 3\% to $15 \%$ in 2012 . This study used an expectation-maximization algorithm to impute these missing values via SPSS 22.0, because of its more accurate estimates than conventional methods under the assumption that the data are missing at random [15].
The final imputed results were highly similar to those from the sample excluding missing values. CFPS was approved by the Institute of Social Science Survey in Peking University. Written informed consent was obtained from all participants.

\subsection{Measurement}

This study used the 20-item CES-D and 8-item CES-D to assess depressive symptoms in 2012 and 2016, respectively. These versions of the scale were chosen because the CFPS collected follow-up information on depression mainly through a short form of the CES-D rather than the full scale. The response options ranged from zero ('rarely or none of the time') to three ('most or all of the time'). Items that described positive affect were reverse coded. The eight items in the short form included depressed affect, such as 'I felt depressed', 'I felt lonely' or 'I felt sad', somatic symptoms, such as 'I felt everything I did was an effort', 'my sleep was restless' or 'I could not get going' and positive affect, such as 'I was happy' and 'I enjoyed life'. The alpha coefficients for the full scale and short-form scale were 0.86 in 2012 and 0.82 in 2016 Higher scores on the CES-D denoted more depressive symptoms.

\subsection{Data Analysis}

Confirmatory factor analysis (CFA) was employed to analyze the factorial structure of the full scale and short form of the CES-D. According to the incongruent findings of the CES-D's latent structure in the literature, three or four competing models were tested. The value of GFI should be above 0.90 and RMSEA below 0.08 for a model that fits well [16]. AIC index was also used to compare models that were not nested, with a lower value representing a better fit.

\section{RESULTS}

The mean score of the full scale of the CES-D was 14.27 in the older Chinese sample. According to the definition of the CES-D, scoring at 16 and above suggests experiencing depressive symptoms. Approximately $33.7 \%$ of the participants suffered from depressive symptoms (data not shown), which is similar to the prevalence of depressive symptoms in Chinese adults in 2012 [3].

Table 1 shows the fit indices for the one-factor, two-factor, three-factor and four-factor models of the full scale and short form of the CES-D. The one-factor CFA model had the worst fit to the data for the full scale, with the largest values of $\chi^{2}$, AIC and RMSEA, and the lowest value of GFI. In the full scale, the model fit of the two-factor model improved substantially, but it was not superior to the other two models. Although the three-factor model had the same GFI and RMSEA values as the four-factor model, the four- 
factor model fitted the data best because of its lower AIC value (2526.67). The results of the difference in $\chi^{2}\left(\chi^{2}\right.$ difference $(\mathrm{df}=3)=13.80, \mathrm{p}<0.01)$ also reflected the two distinct models. The reliability of depressed affect, somatic symptoms, interpersonal problems and positive affect ranged from 0.68 to 0.83 in the full scale.

In the short-form scale, the two-factor and three-factor models fitted the data better than the one-factor model. The three-factor model, the best fitting model, was identified by its lower AIC value and significant difference in $\chi 2$. Cronbach's alpha values of depressed affect, somatic symptoms and positive affect were $0.73,0.60$ and 0.79 , respectively. The standardized factor loadings of all items were significant in both versions of this scale. The standardized loadings of items were greater than 0.40 except the item "Feeling as good as others". The results supported the four-factor model for the full scale and the three-factor model for the eight-item short-form scale.

Table 1. Fit Indices of the CES-D.

\begin{tabular}{|c|c|c|c|c|c|}
\hline Model & $\chi^{2}$ & $\mathrm{df}$ & GFI & AIC & $\overline{\text { RMSEA }}$ \\
\hline \multicolumn{6}{|c|}{ The 20 -item scale in 2012} \\
\hline One factor & 5078.88 & 170 & 0.86 & 5158.88 & 0.09 \\
\hline $\begin{array}{l}\text { Two factor } \\
\text { (depressive symptoms anı } \\
\text { interpersonal problems) }\end{array}$ & 4691.01 & 169 & 0.87 & 4773.01 & 0.08 \\
\hline $\begin{array}{l}\text { Two factor } \\
\text { (depressed and } \\
\text { positive affect) }\end{array}$ & 2832.34 & 169 & 0.92 & 2914.34 & 0.07 \\
\hline $\begin{array}{l}\text { Three factor } \\
\text { (depressed symptoms, } \\
\text { positive affect, and } \\
\text { interpersonal problems) }\end{array}$ & 2448.47 & 167 & 0.93 & 2534.47 & 0.06 \\
\hline Four factor & 2434.67 & 164 & 0.93 & 2526.67 & 0.06 \\
\hline \multicolumn{6}{|c|}{ The 8-item scale in 2016} \\
\hline One factor & 1655.79 & 20 & 0.90 & 1687.79 & 0.15 \\
\hline $\begin{array}{l}\text { Two factor } \\
\text { (depressed and } \\
\text { positive affect) }\end{array}$ & 436.30 & 19 & 0.97 & 470.30 & 0.08 \\
\hline Three factor & 411.13 & 17 & 0.97 & 449.13 & 0.08 \\
\hline
\end{tabular}

\section{DISCUSSION}

\subsection{The Factorial Structure of the CES-D}

The consistency of results from studies using the CES-D in Western countries and in China has been unclear. In the present study, the first analysis examined the factorial structure of the full scale and short scale of the CES-D among the older population in China. The CFA results showed that the four-model factor originally proposed by Radloff [4] has a better fit compared to one-factor, two- factor and three-factor models. The results align with earlier research that analyzed the full scale through the same CFA method [5,6], which supports the first research hypothesis. It is worthwhile to note that the three-factor model, that combines depressed affect and somatic symptoms into a single factor, showed suboptimal model fit. The results reflect that although older adults in China do not blur the distinction between somatic symptoms and depressed affect, some may have a higher presentation of somatic symptoms. Increased age has been shown to be associated with perceived stigma toward depression [17], which may prevent expression of depressed affect.

When comparing findings to other studies in Chinese areas, the alternative models proposed by Cheung and Bagley in Hong Kong [11] and by Han and Jia in a rural area [12] had a weaker model fit in comparison with the four-factor model. Most fit indices of the four-model factor were superior to those of the two-factor model of Cheung and Bagley [11]. Nevertheless, the two-factor model is supported because of poor discriminant validity of the four factors. A local study [12] that identified a three-factor model through EFA but not through CFA cannot lend support to the superiority of this model. The latent structure of the full scale for older people seems to be culturally universal, as most studies involving Caucasian and Chinese samples support the four-factor model [5,6]. The present study supports the factorial structure identified by other scholars, which adds evidence to the psychometric robustness of the CES-D in this age group.

For the latent structure of the eight-item short form of the CES-D with older people, findings support the three-factor model. As such, the second research hypothesis is also supported by data. This is inconsistent with previous work that used EFA and CFA to identify a single factor structure for the short-form scale [13]. When this scale involves three dimensions of depression, such as depressed affect, somatic symptoms and positive affect, the previous study does not compare the one-factor model with alternative models. The CFA of the one-factor model does not have a satisfactory fit to the data (RMSEA $=0.12$ ) until the correlated residuals between the two items on positive affect are established [13]. This implies that positive affect might be distinct from depressed affect. As such, the finding that the one-factor model fits the data better is inconclusive. The one-factor model has a much poorer fit to the data than the two- and three-factor models. The different dimensions of the short-form scale observed in the present study corroborate the extant literature [14]. The results show that the short-form scale, as the full scale, has distinct dimensions of depressive symptoms. Therefore, the short form should be viewed as a similarly valid and reliable instrument to measure depression. In summary, this study examined the psychometric properties of the full scale as well as the short-form scale of the CES-D, which provides the basis for cross-country comparison of measurement invariance for these scales. The factor structure of the full scale of the CES-D in older Chinese adults is as same as that in most Western research. 


\subsection{Limitations}

This study has several limitations. First, although the factorial structure of the full scale of the CES-D is identical to that in most studies, the latent structure of the scale may differ in more traditional cultures, such as India and other East Asian countries. Furthermore, measurement invariance of the CES-D including metric invariance, scalar invariance, and uniqueness invariance between older adults in different countries should be tested in the future.

\section{CONCLUSION}

The four-factor structure for the full CES-D and the threefactor structure for the short-form CES-D have better model fit among older adults in China. The results suggest that acceptable measurement properties of the full- and short-form of the CES-D in Chinese older adults.

\section{REFERENCES}

[1] S.J. Bartels, "Why collaborative care matters for older adults in China," Lancet Psychiat, vol. 2, pp. 286 $287,2015$.

[2] National Bureau of Statistics, China Statistical Yearbook 2020. Beijing: China Statistics Press, 2020.

[3] X. Qin, S. Wang, and C-R Hsieh, "The prevalence of depression and depressive symptoms among adults in China: Estimation based on a National Household Survey," China Eco Rev, vol. 51, pp. 271-282, 2018.

[4] L.S. Radloff, “The CES-D Scale: A self-report depression scale for research in the general population," Appl Psycho Meas, vol. 1, pp. 385-401, 1977.

[5] T.D. Cosco, C.C. Lachance, J.M. Blodgett, B. Stubbs, M. Co, N. Veronese,...and A. W. Prina, "Latent structure of the Centre for Epidemiologic Studies Depression Scale (CES-D) in older adult populations: A systematic review," Aging Ment Health, vol. 24, pp. 700-704, 2020.

[6] B. Zhang, M. Fokkema, P. Cuijpers, J. Li, N. Smits, and A. Beekman, "Measurement invariance of the center for epidemiological studies depression scale (CES-D) among Chinese and dutch elderly." BMC Medical Res Methodol, 2011. DOI: https://doi.org/10.1186/1471-2288-11-74
[7] Y. Chentsova-Dutton, and J.L. Tsai, "Self-focused attention and emotional reactivity: The role of culture," J Pers Soc Psycho, vol. 98, pp. 507-519, 2010.

[8] S. Chokkanathan, and J. Mohanty, "Factor structure of the CES-D scale among older adults in Chennai, India.” Aging Ment Health, vol. 17, pp. 517-525, 2013.

[9] A.G. Ryder, J. Yang, X. Zhu, S. Yao, J. Yi, S.J.Heine, ... and R.M. Bagby, "The cultural shaping of depression: Somatic symptoms in China, psychological symptoms in North America?" J Abnorm Psychol, vol. 117, pp. 300-313, 2008.

[10] J.L. Tsai, B. Knutson, and H.H. Fung, "Cultural variation in affect valuation," J Pers Soc Psycho, vol. 90, pp. 288-307, 2006.

[11] C-K. Cheung, and C. Bagley, "Validating an American scale in Hong Kong: The Centre for Epidemiologic Studies Depression Scale (CES-D)," J Psychol, vol. 132, pp. 169-186, 1998.

[12] M. Han, and C-X Jia, "Reliability and validity of Centerfor Epidemiological Studies Depression scale in different rural populations," Chinese Journal of Public Health, vol. 28, pp. 1265-1267, 2012.

[13] J. Karim, R. Weisz, Z. Bibi, and S. Rehman, "Validation of the eight-item Center for Epidemiologic Studies Depression Scale (CES-D) among older adults," Current Psycho, vol. 34, pp. 681-692, 2015.

[14] C. L. Kimberlin, J.F. Pendergast, D.H. Berardo, and L.C. Mckenzie, "Issues related to using a shortform of the Center for Epidemiological StudiesDepression Scale," Psychol Reports, vol. 83, pp. 411 $421,1998$.

[15] P.D. Allison, "Missing data," In The SAGE handbook of quantitative methods in psychology, R. E. Millsap and A. Maydeu-Olivares, Eds. London: Sage, 2009, pp.72-89.

[16] M.W. Browne, and R. Cudeck, “Alternative ways of assessing model fit," In Testing structural equation models, K.A. Bollen, and J.S. Long, Eds. Beverly Hills: Sage, 1993, pp. 136-162.

[17] F. Yang, B. X. Yang, T.E. Stone, X.Q. Wang, Y. Zhou and S.F. Jiao, "Stigma towards depression in a community-based sample in China," Comprehensive Psychiatry, 2020. DOI:

https://doi.org/10.1016/j.comppsych.2019.152152 